\title{
Analysis of Autopilot Disengagements Occurring During Autonomous Vehicle Testing
}

\author{
Chen Lv, Member, IEEE, Dongpu Cao, Member, IEEE, Yifan Zhao, Daniel J. Auger, Senior Member, IEEE, Mark \\ Sullman, Huaji Wang, Laura Millen Dutka, Lee Skrypchuk, and Alexandros Mouzakitis
}

\begin{abstract}
In present-day highly-automated vehicles, there are occasions when the driving system disengages and the human driver is required to take-over. This is of great importance to a vehicle's safety and ride comfort. In the U.S state of California, the Autonomous Vehicle Testing Regulations require every manufacturer testing autonomous vehicles on public roads to submit an annual report summarizing the disengagements of the technology experienced during testing. On 1 January 2016, seven manufacturers submitted their first disengagement reports: Bosch, Delphi, Google, Nissan, Mercedes-Benz, Volkswagen, and Tesla Motors. This work analyses the data from these disengagement reports with the aim of gaining abetter understanding of the situations in which a driver is required to takeover, as this is potentially useful in improving the Society of Automotive Engineers (SAE) Level 2 and Level 3 automation technologies. Disengagement events from testing are classified into different groups based on attributes and the causes of disengagement are investigated and compared in detail. The mechanisms and time taken for take-over transition occurred in disengagements are studied. Finally, recommendations for OEMs, manufacturers, and government organizations are also discussed.
\end{abstract}

Index Terms-Automated vehicle, Disengagement, humanvehicle interactions, take-over operation, vehicle testing.

\section{INTRODUCTION}

A UTOMATED vehicles have been gaining increasing attention from both academia and industry [1]. The field of automated vehicles is multidisciplinary, involving transportation system, automotive engineering, human factors, information technology, control, robotics, communications, energy, security, and social sciences [2]-[5].

While fully autonomous driving is often considered the "end goal", intermediate "highly automated" vehicles (HAVs) that

Manuscript received February 28, 2017; accepted September 29, 2017. This work was supported by Jaguar Land Rover and the UK-EPSRC grant EP/ N012089/1 as part of the jointly funded Towards Autonomy: Smart and Connected Control (TASCC) Programme. Recommended by Associate Editor Fei-Yue Wang. (Corresponding author: Dongpu Cao.)

Citation: C. Lv, D. P. Cao, Y. F. Zhao, D. J. Auger, M. Sullman, H. J. Wang, L. M. Dutka, L. Skrypchuk, and A. Mouzakitis, "Analysis of autopilot disengagements occurring during autonomous vehicle testing," IEEE/CAA $J$. of Autom. Sinica, vol. 5, no. 1, pp. 58-68, Jan. 2018

C. Lv, D. P. Cao, Y. F. Zhao, D. J. Auger, M. Sullman, and H. J. Wang are with the School of Aerospace, Transport and Manufacturing, Cranfield University, Cranfield, Bedford, MK43 0AL, UK (e-mail: c.lyu@cranfield.ac.uk; d.cao@ cranfield.ac.uk; yifan.zhao@cranfield.ac.uk; d.j.auger@cranfield.ac.uk; m.sullman@cranfield.ac.uk; huaji.wang@cranfield. ac.uk).

L. M. Dutka, L. Skrypchuk, and A. Mouzakitis are with Jaguar Land Rover, Coventry, CV3 4LF, UK (e-mail: lmillen@ jaguarlandrover.com; 1skrypch@jaguarlandrover.com; amouzak1@jaguarlandrover.com).

Color versions of one or more of the figures in this paper are available online at http://ieeexplore.ieee.org.

Digital Object Identifier 10.1109/JAS.2017.7510745 are capable of driving autonomously in some scenarios (but not all) are likely to arrive sooner: HAVs the next few years. In support of this, the USA's National Highway Traffic Safety Administration (NHTSA) issued its "Federal Automated Vehicles Policy" in September, 2016. In this document, the NHTSA adopted the approach of SAE for describing levels of vehicle automation. The SAE definitions divide vehicles into six levels based on "who does what, when" [6], [7]. SAE Level 1 corresponds to "driver assistance" systems; SAE Level 4 describes vehicles with "high automation" with the ability to manage safety-critical functions such as steering, accelerating and braking.SAE Level 2 ("partial automation") and Level 3 ("conditional automation") represent the transitional region between driver assistance and high automation, and they are a significant focus of emerging work.

In Level 2 and Level 3 automation, the driver cedes longitudinal and lateral control of the vehicle to a varying degrees [8].When there are driving tasks that the vehicle's automation systems can no longer handle, the automated control mode disengages and control authority is given back to the driver. The disengagement process, along with the take-over (TO) operation, is of key importance and greatly affects automated vehicle's safety and comfort. To address these challenges in driver-vehicle interactions at Level 2 and Level 3 automation, many researchers have explored advanced driver assistance systems (ADAS) and human-machine interfaces (HMIs) from a variety of viewpoints [9]-[12]. However, disengagement events and their associated take-over events have rarely been investigated in real vehicle testing scenarios.

The US state of California is noted for its encouragement of autonomous vehicle technology and it allows manufacturers to perform testing on public roads. California's Autonomous Vehicle Testing Regulations require every manufacturer testing vehicles on the state's public roads to submit an annual report summarizing the disengagements experienced during testing. These disengagement reports are due by January 1 of each year. Seven manufacturers, namely Bosch, Delphi, Google, Nissan, Mercedes-Benz, Volkswagen, and Tesla Motors submitted their first disengagement reports on January 1, 2016 [13]-[19]. To better understand the mechanisms of driver-vehicle interactions during the disengagement process, and to improve the Level 2 and Level 3 automation technology, this paper reviews the seven publicly reported disengagement files. The disengagement events with their various causes are investigated and compared in detail. Take-over mechanism and time are also examined through the vehicle tests. These findings shed light on the refinement of automated technology 
and driver-automation collaboration.

To present the details, the rest of this paper is arranged as follows: Section II defines and classifies the disengagement events focused on in this study, and presents the overall conditions of the disengagement reported by manufacturers; Section III analyses the main reasons of these disengagements; Section IV discusses the disengagement cases of stage-I and stage-II manufacturers, respectively; Section V investigates the take-over mechanism and time reported in the disengagements; Section VI proposes recommendations to original equipment manufacturers (OEMs), manufacturers, and government organizations, respectively, and discusses the opening challenges associated with driver-vehicle interactions; Section VII concludes the findings and discusses possible future work.

\section{Disengagement OvervieW}

\section{A. Definition of Disengagement}

The California Department of Motor Vehicles (DMV) rule defines the disengagement which needs to be reported as a deactivation of the autonomous mode when a failure of the autonomous technology is detected, or when the safe operation of the vehicle requires that the autonomous vehicle test driver disengages autonomous mode and takes immediate manual control of the vehicle [20].

Remark 1: The above definition is necessary to ensure that manufacturers are not reporting every common or routine disengagement. And since these reported disengagements refer to those immediate ones, the automated technology where disengagements events occurred corresponds to SAE Level 2 automation, rather than Level 3 (Level 3 automation is able to give driver a sufficiently comfortable transition time to take over the manual control).

\section{B. Classification of Disengagement}

The above defined disengagements of autonomous mode can be divided into:

1) Passive disengagement $(P D E)$ : When a failure of the autonomous technology is detected, disengagement is required by the automation system, and the driver undertakes an immediate take-over.

2) Active disengagement $(A D E)$ : Automation system does not recognize any failure, but the driver monitors the situation and actively takes an immediate manual control of the vehicle, disengaging the autonomous mode.

\section{Overview of Automated Testing and Disengagements}

An overview of the number of disengagements, with the number of automated driving miles for the seven manufacturers (represented as A-G), are listed below.

1) Manufacturer A reported 341 disengagements in 424331 miles driven [15];

2) Manufacturer B reported 405 disengagements in 16662 miles driven [14];

3) Manufacturer $\mathrm{C}$ reported 261 disengagements in 14945 miles driven [18];

4) Manufacturer D reported 1031 disengagements in 1739 miles driven [17];

5) Manufacturer E reported 106 disengagements in 1485 miles driven [16];

6) Manufacturer $F$ reported 625 disengagements in 935 miles driven [13];

7) Manufacturer $G$ reported zero disengagements (No testing on public streets) [19].

Based on the results in Fig. 1, Company A is along way ahead in terms of autonomous vehicle testing miles on public roads. Detailed data can be found in Table I. As the automated driving technology being developed and refined constantly, for most of these manufacturers, the number of disengagements occurring each month is steadily decreasing over time, as shown in Fig. 2. The number of these disengagements, each month, by company can be found in Table II.

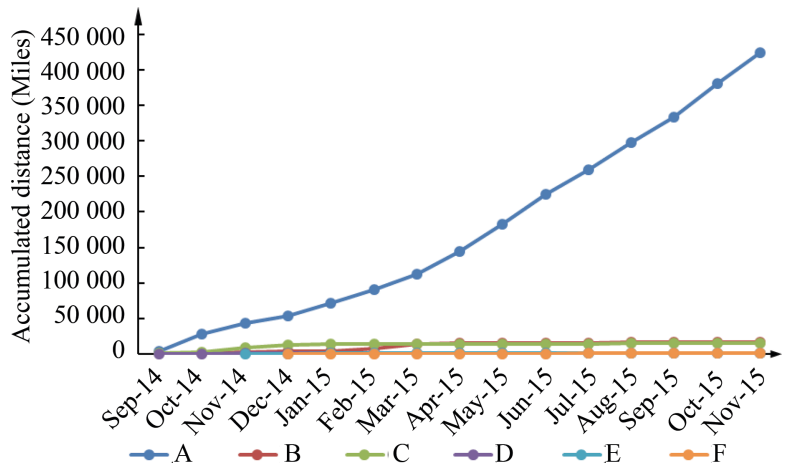

Fig. 1. Automated driving miles on public roads for the six companies.

The environments that the automated cars operate are very important. Mastering autonomous driving in various conditions, including different locations, weather, and multi-type road surfaces, requires the automated technology to be smart and robust enough to handle all possible road circumstances [21], [22].

1) Locations: Street, Interstate way, freeway/highway; rural road, parking facility.

TABLE I

Reported Automated Testing Miles on Public Roads

\begin{tabular}{|c|c|c|c|c|c|c|c|c|c|c|c|c|c|c|c|c|}
\hline $\mathrm{mpa}$ & Sep-14 & Oct-14 & Nov-14 & Dec-14 & Jan-15 & Feb-15 & Mar-15 & Apr-15 & May-15 & Jun-15 & Jul-15 & Aug-15 & Sep-15 & Oct-15 & Nov-15 & Total \\
\hline A & 4207.2 & 23971.1 & 15836.6 & 9413.1 & 18192.1 & 18745.1 & 22204.2 & 31927.3 & 38016.8 & 42046.6 & 34805.1 & 38219.8 & 36326.6 & 47143.5 & 43275.9 & 424331 \\
\hline B & & & & & & & - & & & & & & & & & \\
\hline $\mathrm{C}$ & 1026.5 & 910.3 & 6405.09 & 4375.7 & 1312.33 & 160.94 & 12 & 3. & 99. & & 77 & 32 & 12 & $\mathrm{~N}$ & N/A & 14945.84 \\
\hline D & N/A & 1291 & 1164 & 1 & & 3 & 6604 & & 8 & & & 63 & & & 48 & 1739.08 \\
\hline $\mathrm{E}$ & 11 & & & & & & $\mathrm{~N} /$ & 131 & & & 91 & 108 & 93 & 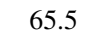 & 34.6 & 1485.4 \\
\hline $\mathrm{F}$ & 107.65 & 288 & 706.46 & 42.81 & 29.43 & 38.9 & 18.3 & 47 & 55.1 & 171.63 & 119.82 & 22.17 & 18.72 & 29.59 & 43.5 & 935.1 \\
\hline
\end{tabular}


TABLE II

Reported Number of Disengagements during Automated Testing

\begin{tabular}{|c|c|c|c|c|c|c|c|c|c|c|c|c|c|c|c|c|}
\hline Company & Sep-14 & Oct-14 & Nov-14 & Dec-14 & Jan-15 & Feb-15 & Mar-15 & Apr-15 & May-15 & Jun-15 & Jul-15 & Aug-15 & Sep-15 & Oct-15 & Nov-15 & Total \\
\hline A & 2 & 19 & 21 & 43 & 53 & 14 & 30 & 51 & 13 & 11 & 29 & 7 & 16 & 16 & 16 & 341 \\
\hline B & N/A & 39 & 43 & 107 & 35 & 10 & 79 & 11 & 6 & 9 & 13 & 7 & 6 & 12 & 28 & 405 \\
\hline $\mathrm{C}$ & 18 & 68 & 101 & 56 & 15 & 1 & 0 & 0 & 0 & 1 & 0 & 0 & 1 & N/A & N/A & 261 \\
\hline D & 44 & 244 & 189 & 36 & 48 & 16 & 39 & 72 & 34 & 127 & 69 & 24 & 50 & 17 & 22 & 1031 \\
\hline E & N/A & N/A & 7 & 32 & 31 & 0 & 0 & N/A & N/A & N/A & N/A & N/A & N/A & 28 & 8 & 106 \\
\hline $\mathrm{F}$ & N/A & 0 & 0 & 126 & 86 & 21 & 0 & 83 & 10 & 0 & 40 & 35 & 27 & 103 & 94 & 625 \\
\hline
\end{tabular}

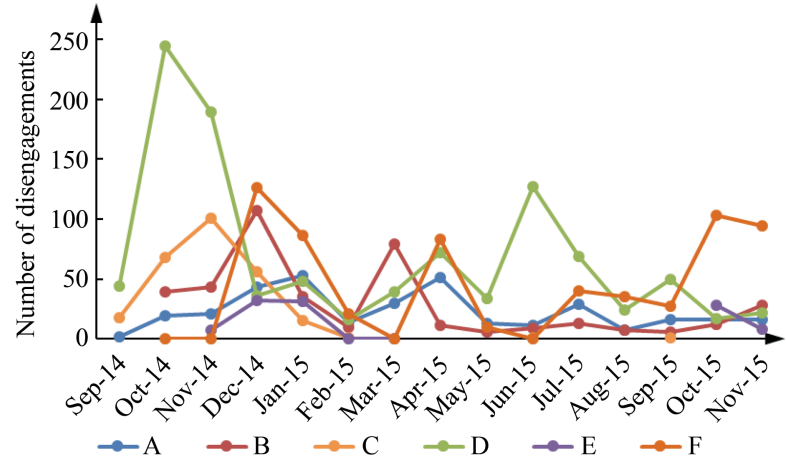

Fig. 2. Number of disengagements by month.

2) Weather: Sunny, cloudy, rainy, clear (night) [16].

3) Road surface conditions: Dry, wet, poor road conditions with holes or bumps [17], poor lane markings, such as faded markings, and freshly paved roadway [14].

In order to comprehensively evaluate automated driving miles and disengagements, "miles per disengagement (MPD)" is adopted as an indicator to evaluate the maturity of the autonomous technology. MPD is defined as follows.

$$
M P D=\frac{S}{n}
$$

where $S$ is the automated driving miles during testing, and $n$ is the number of disengagements occurred during testing.

According to Fig. 3, at the end of 2015 the MPD of Company A remains stable at around 3000, while other companies' MPDs were typically within 100. Based on the MPD value, we define two stages to indicate different maturities of Level 2 automation technology.

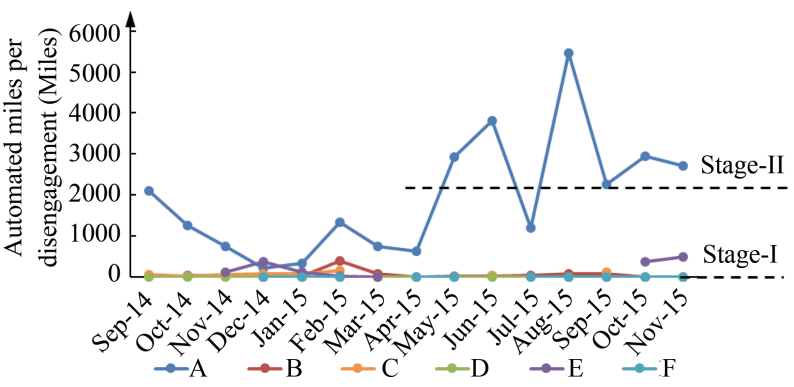

Fig. 3. Automated miles per disengagement of the six manufacturers.

Stage 1: MPD below 2000, indicating the initial stage of Level 2 automation, with a lot of fundamental functions of automated system needing to be refined and improved.

Stage 2: MPD above 2000, corresponding to the advanced level with matured Level 2 automation system, approaching Level 3 automation.
Thus, the six manufacturers covered in this report can be clearly classified into two groups with different stages of maturity in automated driving technology.

1) Stage-I manufacturers: B, C, D, E, and F;

2) Stage-II manufacturer: A.

\section{Main Reasons for Disengagements}

Many factors can impact autonomous control performance, and result in disengagements. These reasons include (but are not limited to) those listed in Table III and Fig. 4.

TABLE III

CAUSES OF THE DIFFERENT TYPES OF DISENGAGEMENTS

\begin{tabular}{cc}
\hline PDE & ADE \\
\hline Hardware issues & Software limitations \\
Software failures & Hardware issues \\
Weather conditions & Emergencies \\
Road surface conditions & Precautionary intervention \\
\hline
\end{tabular}

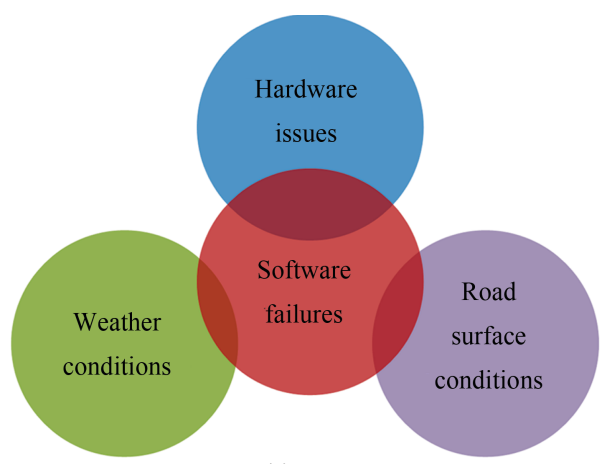

(a) PDE

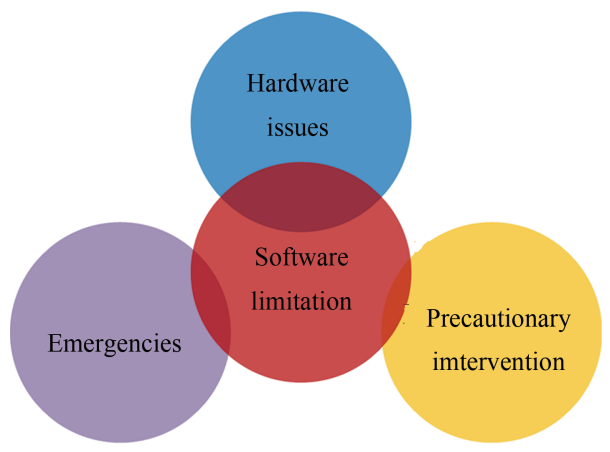

(b) ADE

Fig. 4. Main causes of PDEs and ADEs.

A. Typical causes of PDE

1) Hardware issues 
Hardware discrepancy indicates that a hardware element failed or it is not performing as expected. Some typical reported causes have been listed as follows.

A failure occurred in the vehicle controller [16];

Damage to sensors, wires, actuators, and other physical devices.

\section{2) Software issues}

Software discrepancy covers inadequacies, involving issues of environment perception, object recognition, vehicle positioning and localization, decision making, path planning, trajectory generation, and vehicle control. Some typical causes reported are:

Perceiving overhanging branches as an obstacle [15];

Another vehicle approached from the side but was undetected by the perception system [16];

The recognition system lost the trajectory of the preceding vehicle [16];

Generation of route goal failed [16];

Localization of the automated vehicle failed [16];

Position estimation logic failure caused the automated vehicle to begin traveling outside of its lane [16];

Departure logic failed, and the automated vehicle would not begin moving [16];

Problems with controller area network (CAN) bus data rate and data transmission causing the automated control to shut off [16];

Watchdog error;

System tuning and calibration [16].

3) Weather conditions

Problems with weather conditions during testing caused the autonomous controller to shut off.

Traffic light detection fault due to poor lighting conditions [16];

Object detection failure due to sun glare, rain, or twilight [17];

Failures with poor visibility due to heavy rain, snow, fog, etc.;

Failures with bright light, such as oncoming headlights or direct sunlight;

Failures due to extremely hot or cold temperatures.

\section{4) Road surface conditions}

Problems causing autonomous control to shut off due to poor road surface conditions.

Road with holes or bumps [17];

Poor lane markings, such as faded markings, and freshly paved roadway [14].

\section{B. Typical causes of $A D E$}

Typical causes of ADE include software limitation, hardware issues, emergencies, and precautionary interventions. Detailed descriptions and related example cases are listed as follows.

\section{1) Software limitations}

Although there was no failure detected, the automated system was unable to handle high-stage tasks in complex situations, or object perceptions, vehicle's trajectories, maneuvers, and behaviors made by the automated system were undesirable due to the limitation of software, resulting in an active takeover. Some typical cases are:

Complete lane change in heavy traffic [14];

Too many pedestrians and vehicles at the intersection for the system to predictably handle [16];

The automated vehicle moved uncomfortably close to a parked car [15];

The automated vehicle drove too close to the left or right side of the lane [16];

The automated vehicle did not recognize a vehicle that stopped in front of it and failed to slow down [16];

A vehicle pulling out of a parking lot was not recognized by the automated vehicle perception system [16];

System incorrectly recognized the preceding vehicle as a vehicle in the next lane [16];

The automated vehicle began to merge into a lane behind another vehicle very closely [16];

A vehicle stopped suddenly in front of the automated vehicle at an intersection [16];

The automated vehicle was slowly encroaching on the preceding vehicle [16];

The driver felt a delay in deceleration, so the driver took over the brake operation [16];

The automated vehicle did not enter the correct lane [16].

2) Hardware issues

Hardware issues that made the driver feel to immediately take over manual control of the vehicle.

\section{3) Emergencies}

Emergency situations that make the driver actively take over the control authority of the vehicle from the automation system for safety reasons. Typical cases include:

Emergency vehicles [14], [15] (To solve this issue, recently Google proposed a system and method for detecting and responding to emergency vehicles [23]);

Accidents or collisions [13];

Unexpected or reckless behaviors from other vehicles. For example: While the automated vehicle was merging autonomously, another vehicle approached in front of the automated vehicle suddenly, and it was undetected by the perception system; Another vehicle was about to rear-end the automated vehicle due to sudden deceleration of the automated vehicle.

\section{4) Precautionary intervention}

Problems causing autonomous control to shut off due to poor road surface conditions.

To avoid construction zones [13]-[17];

To give extra space for a cyclist [14];

Precautionary intervention due to pedestrian traffic [14];

To ensure vehicle safety in bad weather conditions [17].

Remark 2: The above does not represent an exhaustive list of situations that may interfere with proper operation of automated control. In the initial stages of automated driving (e.g., Level 1 and Level 2 automation), operators should never completely depend on these components to remain safe. It is the driver's responsibility to stay alert, and be ready to take control of the vehicle at all times. 


\section{CASE Study of Manufacturers}

\section{A. Analyses of Stage-II Disengagement (DE) Cases}

As a leading company, the Stage-II manufacturer A's disengagement cases are presented in order to analyze the development course of the automated driving technology.

1) An overview: The numbers of the Stage-II manufacturer's PDE and ADE cases in each month have been listed in Fig. 5. According to the data, PDEs dominate the disengagement cases. After experiencing a significant increase with almost 50 cases per month before May 2015, the amount of PDEs start to decrease considerably, and remains table at below 20 per month, indicating that the automation control system has been gradually refined. In contrast, the number of ADEs remains stable with less than 10 cases per month.

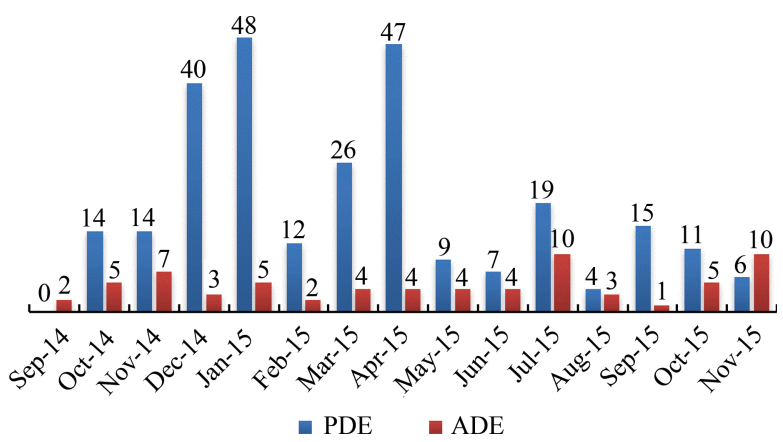

Fig. 5. Numbers of PDE and ADE for Company A each month.

Fig. 6 displays how the number of autonomous miles driven between disengagements has changed over time. In general, the number of autonomous miles between each disengagement is increasing steadily. The rate of DE has dropped from 744 miles per disengagement in Q4 of 2014 to over 2800 miles per disengagement in Q4 of 2015. Specifically, the rates of PDE and ADE have dropped from 1026 miles and 3398 miles per disengagement in the Q4 of 2014 to 5749 miles and 6878 miles per disengagement in Q4 of 2015, respectively. These also demonstrate the gradual improvements and maturity of the automated driving technology that company A achieved during this period.

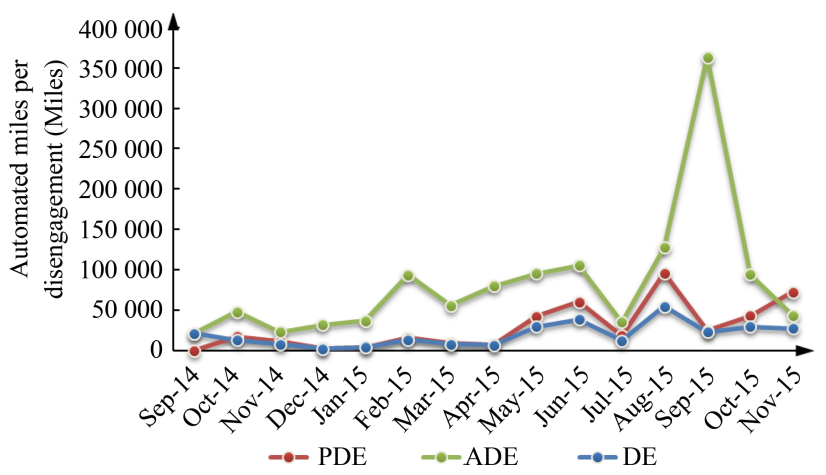

Fig. 6. Automated miles per disengagement for Company A each month.

2) PDE of Stage-II Manufacturer's Cases: Taking a detailed look at the data, the main reasons causing PDEs can be placed into four categories, namely: hardware issues, software failures, weather conditions, and road surface conditions. As shown in Fig. 7, software failures dominate the PDE causes each month. These software issues include initial stage problems of perception, decision making, and control, corresponding to Level 1 and Level 2 automation functions. However, the number of PDEs decreases significantly following April 2015, indicating the continuous refinement and development of the software.

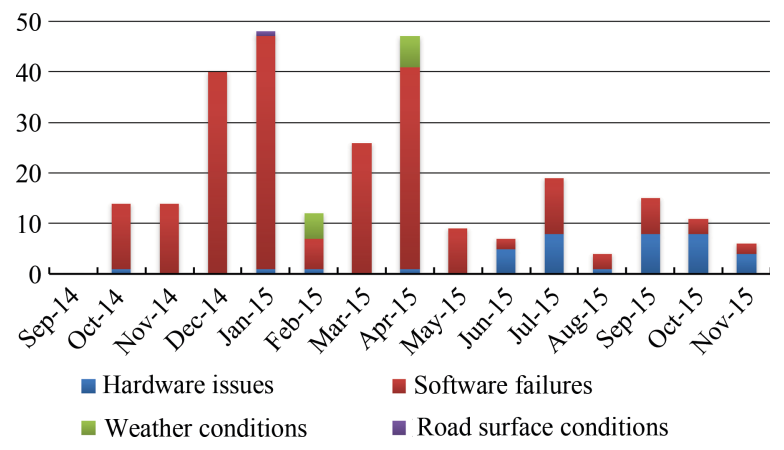

Fig. 7. Company A's PDEs causes by month.

According to Fig. 8, it can be clearly seen that the software problems take up over $80 \%$ of the proportion among the four PDE reasons for failure during the entire testing process. Furthermore, the proportion of hardware failures, poor weather and road conditions are $13.97 \%, 4.04 \%$, and $0.37 \%$, respectively.

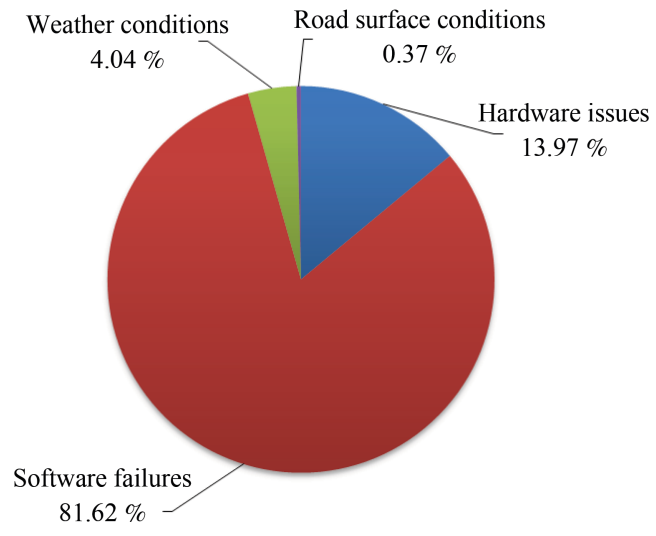

Fig. 8. Proportion of different causes of Company A's PDEs.

3) ADE of Stage-II manufacturer's cases: The main causes of ADE failures were software limitations, hardware issues, emergencies, and precautionary interventions, respectively.

As shown in Fig. 9, it is software limitations that dominate the causes of ADE each month. The number of ADEs caused by software problems has not obviously decreased during this period of time. This is because that these ADE

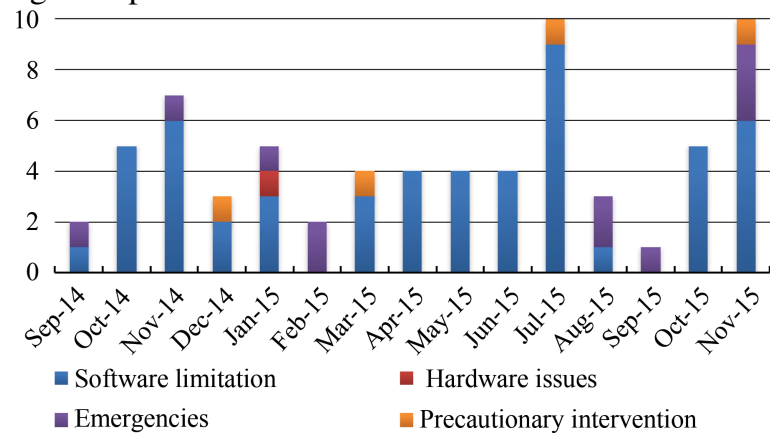

Fig. 9. Company A's ADEs causes by month. 
software problems usually correspond to Level 3 Automation functions, which is much more difficult to improve. Besides this, the essence of disengagements caused by emergencies and precautionary interventions are also closely related to software limitations. Since the automation technology is not robust and intelligent enough so far, the software lack capability to handle advanced driving tasks in complex situations, such as tackling emergencies. Thus, the human operators still do not fully trust the automation control, and they intervene in some cases to guarantee safety.

Fig. 10 shows that software problems account for over $75 \%$ of ADEs'. Combining software failures, emergencies and precautionary interventions together account for $98 \%$ of $\mathrm{ADE}$ cases.

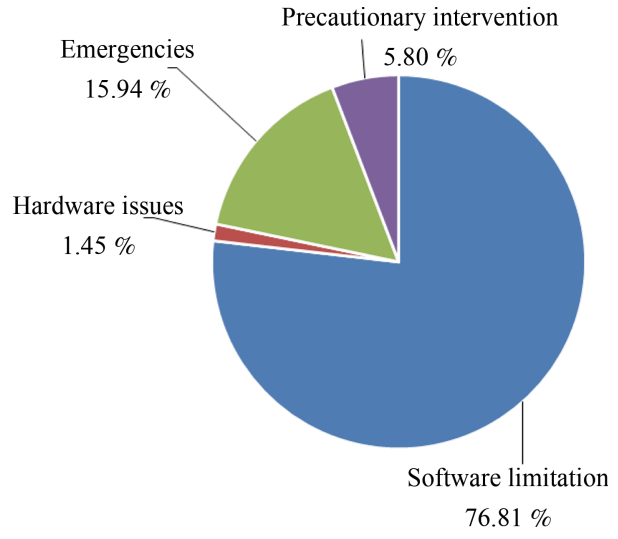

Fig. 10. Proportion of each cause of Company A's ADEs.

\section{B. Detailed Analysis of Stage-I Manufacturers' DE Cases}

The numbers of disengagement events for the five StageI companies manufactures are analysed together, in order to find some commonalities and characteristics based on a larger dataset.

1) An overview: Fig. 11 shows that PDE dominates the disengagement cases in each month from Q4 of 2014 to Q1 of 2015. However, since Jan 2015, the absolute number of PDEs steadily decreases to within 50 per month at the end of the year.

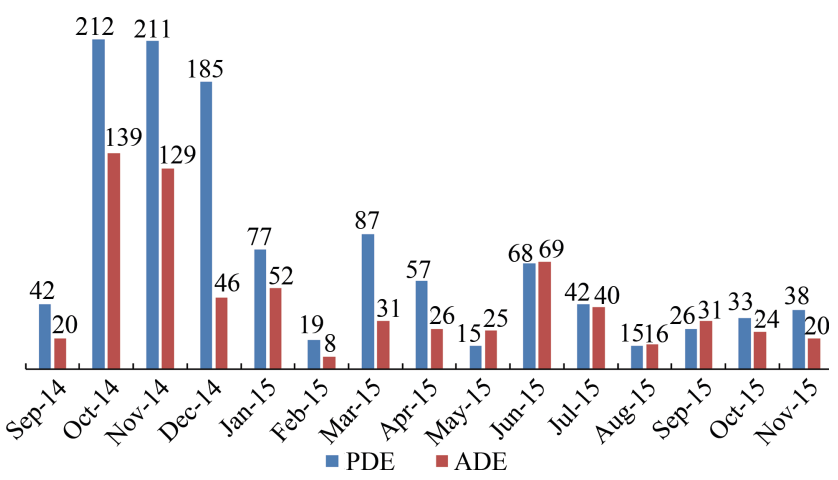

Fig. 11. Number of PDE and ADE of Stage-I companies by month.

However, considering the distance driven, as shown in Fig. 12, the overall automated driving miles of these five Stage-I companies are less than 1/10 of Company A's total autonomous driving miles on public roads. Therefore, number of autonomous miles between disengagements is only around
10 miles (Company A: around 2800 miles), which clearly shows their immaturity in automated driving technology, and also indicates the importance of on-road testing for the development of highly automated vehicles.

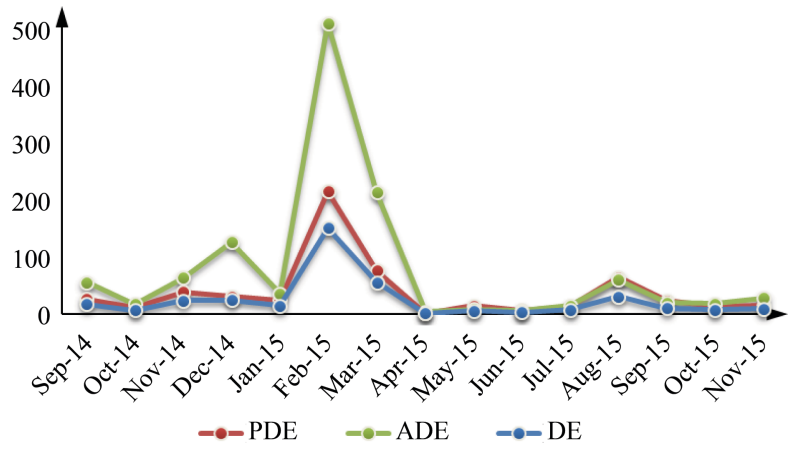

Fig. 12. Automated miles per disengagement of Stage-I companies by month.

2) PDE of Stage-I manufacturers' cases: The reasons for PDEs among Stage-I manufacturers are also due to hardware issues, software failures, weather conditions, and road surface conditions. As shown in Fig. 13, the most common cause was software failures, but the other three causes always contributed to the number of PDEs.

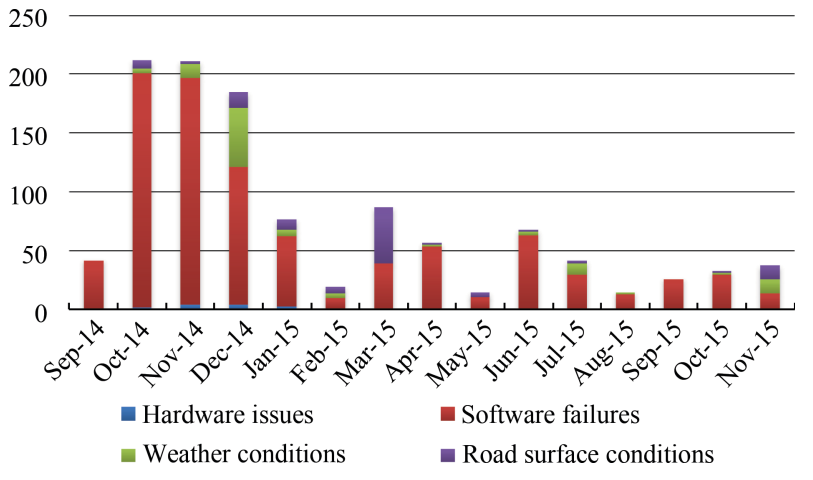

Fig. 13. PDE causes for Stage-I companies by month.

Based on the pie chart in Fig. 14, weather conditions and road surface conditions account for over 9\% of PDE cases, indicating a deficiency in the Level 2 and Level 3 automation functions of these Stage-I companies.

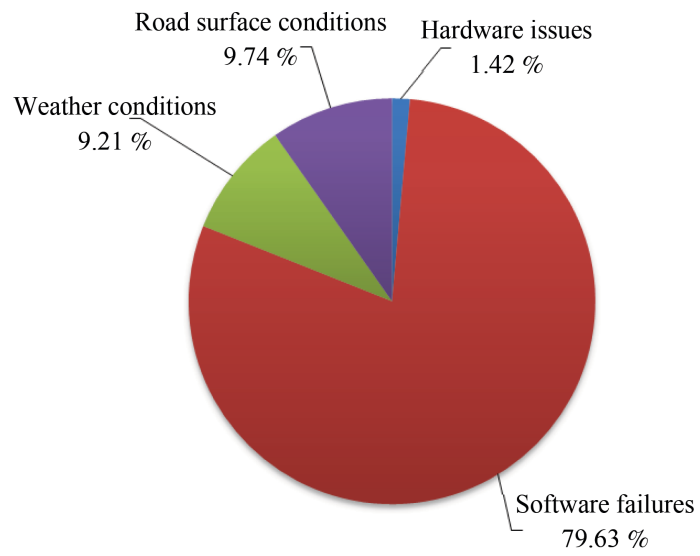

Fig. 14. Proportion of PDEs causes for Stage-I companies. 
3) ADE of Stage-I manufacturers' cases: Based on the data shown in Fig. 15, similar to Company A, software failures were the most common causes of ADE in Stage-I manufacturers. As previously mentioned, the disengagements caused by emergencies and precautionary intervention are actually closely related to software failures.

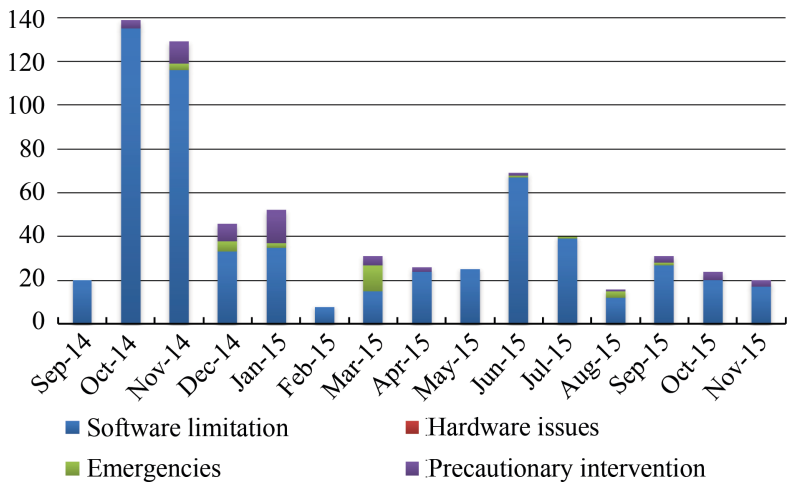

Fig. 15. Causes of ADEs for Stage-I companies' by month.

For Stage-I companies, which three causes account for almost $100 \%$ of the ADEs (see Fig. 16). This phenomenon shows the difficulty in developing high level automation technology, and also indicates the huge gap between their current status and the high automation target of the Stage-I players.

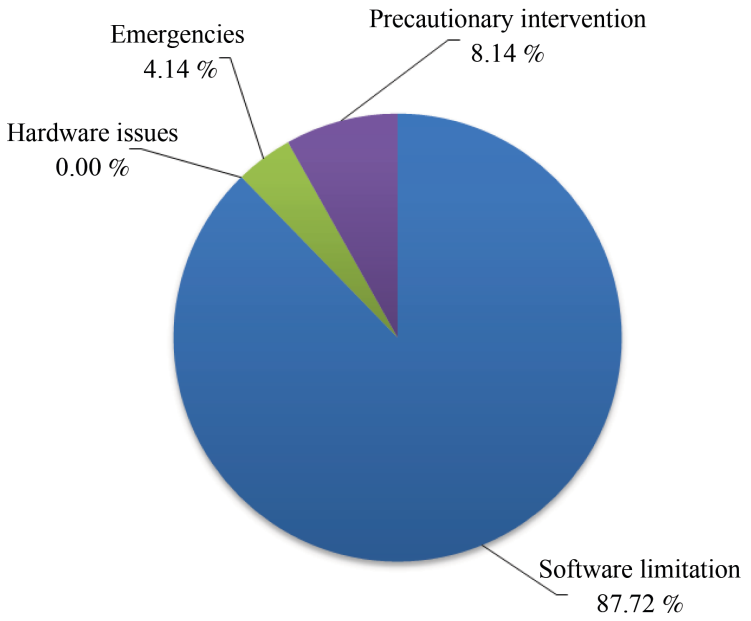

Fig. 16. Proportion of different causes of Stage-I companies' ADEs.

Remark 3: According to the above analysis, the software issues or limitations are the main causes of the disengagements. To improve and refine the software, manufacturers and other entities should follow guidance, best practices, design principles, and standards developed by established standardization organizations such as the International Standards Organization (ISO) and SAE International [24]. The NHTSA also pointed out that the automotive industry should monitor the evolution, implementation, and safety assessment of artificial intelligence (AI), machine learning, and other relevant software technologies and algorithms in order to improve the effectiveness and safety of HAVs [17].

\section{TaKe-Over Mechanism and Time}

As long as there are situations that cannot be handled by automation, the driver has to be available to take over the driving task in a safe manner. Research on the mechanism and elapsed time of the take-over actions can help us to better understand at which point a driver's attention must be directed back to the driving task, and to synthesize suitable shifting control algorithms.

\section{A. Take-Over Mechanism}

A take-over action can either be requested by the automation system or directly triggered by manual input from driver or operator.

1) Take-over request: The take-over request is usually alerted through distinct audio and/or visual feedback after failures have been detected, indicating that immediate manual control is needed by the driver.

2) Take-over operation: After receiving the take-over request alerted by the automation system, or when the driver wants to manually control the vehicle, the take-over transition can be triggered by driver through actions, including pressing the auto/manual switch, or manipulating the steering wheel, brake or accelerator. The take-over processes of each manufacturer are outlined below.

a) Manufacturer A: the test driver is given a distinct audio and visual signal, indicating that immediate take-over is required [15].

b) Manufacturer B: any hardware failure triggers a buzzer to alert the vehicle operator that he/she needs to take over. The vehicle operator always has one hand on the steering wheel and one hand on the auto/manual toggle switch on the vehicle's central console. Pressing the auto/manual switch kills all power to the automated system actuators (throttle, steering, brakes, and shifter) and allows the operator to instantaneously take full control [14].

c) Manufacturer E: test driver is alerted to technology failure, or the driver actively overrides the system with manual brake/accelerator/steering input, causing the controller to disengage [16].

d) Manufacturer F: when a disengagement from automated mode occurs, the driver is immediately alerted audibly and visually and then immediately reassumes control. However, reassuming control does not necessarily mean the driver must apply an immediate, measurable input on the steering wheel, brake or gas pedal or any other input device [13].

e) Manufacturer G: The warning is designed to provide both visual and audible alerts. The automated system does not attempt to apply the brakes or decelerate the vehicle. When seeing and/or hearing a warning, it is the driver's responsibility to take corrective action immediately. Disengaging automated control can be realized by pressing the brake or briefly pushing the cruise control lever away from the driver [25].

\section{B. Take-Over Time}

In general, the take-over time can be defined as the period of time elapsed from when the driver was alerted of a technology failure to when the driver assumed manual control of the vehicle [15]. Currently there is no standard methodology for measuring the take-over time. Furthermore, manufacturers' reports did not adequately describe their methods for logging 
this data. Take-Over elapse time reported in the disengagement events of each manufacturer is reported in Table IV.

TABLE IV

TAKE-OVER TIME REPORTED IN DISENGAGEMENTS OF EACH MANUFACTURER

\begin{tabular}{cccccccc}
\hline \multicolumn{2}{c}{ TO time } & \multicolumn{7}{c}{ Company } \\
& & $\mathrm{A}$ & $\mathrm{B}$ & $\mathrm{C}$ & $\mathrm{D}$ & $\mathrm{E}$ & $\mathrm{F}$ \\
\hline \multirow{3}{*}{$\mathrm{PDE}$} & $\max$ & $2.20 \mathrm{~s}$ & $<1.00 \mathrm{~s}$ & $21.00 \mathrm{~s}$ & $7.95 \mathrm{~s}$ & $4.00 \mathrm{~s}$ & N/A \\
& $\min$ & $0.20 \mathrm{~s}$ & $<1.00 \mathrm{~s}$ & $0.00 \mathrm{~s}$ & $0.00 \mathrm{~s}$ & $<1.00 \mathrm{~s}$ & N/A \\
& avg. & $0.84 \mathrm{~s}$ & $<1.00 \mathrm{~s}$ & $3.06 \mathrm{~s}^{*}$ & $0.86 \mathrm{~s}$ & $<1.00 \mathrm{~s}$ & N/A \\
& ADE & N/A & N/A & N/A & N/A & $0 \mathrm{~s}$ & N/A \\
\hline
\end{tabular}

* In calculating the average take-over time of Manufacturer C's DEs, three extremely large recorded data, namely $29 \mathrm{~s}, 737 \mathrm{~s}$, and $14284 \mathrm{~s}$, were considered to be outliers and excluded [18].

1) Manufacturer A: Our test drivers are trained and prepared for these events and the average driver response time for all measurable events was $0.84 \mathrm{~s}$ [15].

2) Manufacturer B: In all cases that have a record of the disengagement time, this time was measured as $<1 \mathrm{~s}$ (no detailed duration). In all remaining cases the disengagement time was recorded as N/A [14].

3) Manufacturer $C$ : The average take-over time was $3.06 \mathrm{~s}$ [18].

4) Manufacturer D: In general, the average recorded reaction time in disengagement cases was $0.875 \mathrm{~s}$. However, according to the details of their report, only automatic disengagements recorded the reaction time, while the reaction time of disengagements was not provided [19].

5) Manufacturer E: Generally less than 1s on average. In all the driver over ride cases, the time was recorded as $0 \mathrm{~s}$; in almost all the cases of automation system fails, the time was recorded as $<1 \mathrm{~s}$. In one exception the elapsed time was $4 \mathrm{~s}$.

6) Manufacturer $F$ : We are unable to measure the time period for every single disengagement because not all driving situations require measurable driver input. Our test vehicle safety approach, therefore, includes the process of transition from automated to manual mode in a specific test driver training. Only trained drivers are allowed to operate a test vehicle and they constantly monitor the vehicle's operation. This safety approach has been reviewed by an independent 3rd party safety organization [13].

Remark 4: Although most of the average values of the reported take-over time are within $1 \mathrm{~s}$, a take-over transition is never an easy task that can be rapidly and easily completed. In all reported automated vehicle tests the test drivers were well-trained and experienced ones, who were concentrating and prepared to take over the vehicle's control. However, in daily life driving, we are unable to guarantee all the drivers are well trained and concentrate enough to resume the manual control so quickly. Furthermore, the take-over performance is closely related to driving scenarios, driver's workload, attention, drowsiness, whether they have situational awareness, etc. Thus, how to accurately detect driver's behaviors and attention, and the design of the HMI remain important challenges that require exploring. The NHTSA also pointed out that manufacturers and other entities should consider whether it is reasonable and appropriate to incorporate driver engagement monitoring into Level $3 \mathrm{HAV}$ systems [17].

\section{DISCUSSION AND RECOMMENDATIONS}

Disengagement is a key indicator reflecting the maturity of the automated driving technology. Based on the above analyses, we believe that different types of disengagements correspond to distinguished maturity stages of the developed automation technology.

As shown in Fig. 17, PDE and ADE fit the characteristics in between Level 2 and Level 3 automation. Specifically, PDE corresponds to the initial development stage of Level 2. Adequately resolving PDE failures will lead to the manufacturer reaching the maturity-I stage of Level 2 automation. Beyond PDE, those ADE cases can be mapped into the higher stages of Level 2. The causes of ADEs usually indicate more difficult to solve problems of the automated functions. If the ADEs' issues can be well handled, the technology is almost reaching Level 3 automation.

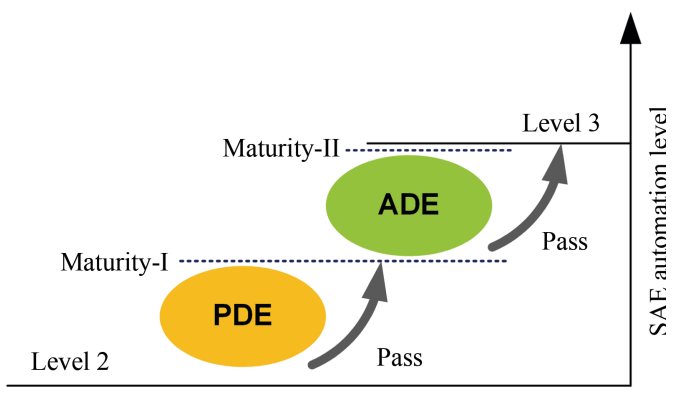

Fig. 17. Relations between reported disengagements and automation levels.

\section{A. Recommendations}

Based on the above analysis, in order to help develop and refine automated driving technologies, especially the Level 2 and Level 3 automation technologies, some recommendations for OEMs, manufacturers, and government organizations are provided below.

1) For OEMs: Disengagement with driver take-over is a complex process which is crucial to driving safety and ride comfort during automated driving. According to the analysis of disengagement data, the dominant causes for both PDE and ADE are software issues, which cover the inadequacies of perception, decision making, path planning, and vehicle control. Heading toward higher automation, the performance and robustness of the software needs to be improved urgently. OEMs should follow a robust software design and validation process based on a systems-engineering approach with the goal of designing HAV systems free of unreasonable safety risks. Also as the NHTSA recommended, OEMs should monitor the evolution, implementation, and safety assessment of artificial intelligence, machine learning, and other relevant software technologies and algorithms to improve the effectiveness and safety of HAVs [6].

In addition, understanding the interaction between the vehicle and the driver is of great importance. Especially in systems of SAE Level 2 and Level 3, human drivers are expected to return to the driving tasks and take over driving 
responsibilities, but drivers' ability to do so is limited by human capacity to stay vigilant when disengaged from the driving task. OEMs should consider how to incorporate driver attention, inattention, and engagement monitoring into automated systems. Furthermore, how HAVs will signal intentions to the environment around the vehicle, including pedestrians, bicyclists, and other vehicles, these factors should also be considered by OEMs documented processes for the assessment, testing, and validation of the vehicle HMI are needed.

2) For manufacturers: For integrating all the components in an automated vehicle, manufacturers should suitably determine their system's AV level in conformity with SAE International's published definitions. For all HAV systems, the manufacturer should address the cross-cutting items as a vehicle or equipment is designed and developed to ensure that HMI design best practices have been followed; that appropriate crash/occupant protection has been designed into the vehicle; and that consumer education and training have been addressed [6].

Especially for handling the disengagements with human driver take-overs, manufacturers should have a documented process for transitioning to a minimal risk condition when a problem is encountered. HAVs operating on the road should be capable of detecting HAV system issues, and informing the human driver in a way that enables the driver to regain adequate control of the vehicle as swiftly and safely as possible. Fall back strategies should take into account that human drivers may be inattentive, under the influence of alcohol or other substances, drowsy, or physically impaired in some other manner. The fall back actions should be administered in a manner that will facilitate safe operations of the vehicle and minimize erratic driving behavior, and should also minimize the effects of errors in human driver recognition and decisionmaking during and after transitions to manual control. Besides this, disengagement events should also be well analysed and utilized after happening. A good example is given by Google. They employs a powerful simulator that allows them to "replay" each disengagement with the behavior of the vehicle as well as the behavior and positions of other road users in the vicinity [15].

3) For government organizations: Government organizations play an important role in facilitating automated vehicles, such as ensuring they are safely deployed, and promoting their life-saving features.

To help develop and improve automated technology, governments should retain their traditional responsibilities for vehicle licensing and registration, traffic laws and enforcement, and motor vehicle insurance and liability regimes for automated vehicles. More testing areas and facilities should be established and upgraded for supporting the development of automated technology [6].

Adequate education and training is imperative to ensure safe deployment of automated vehicles. Apart from manufacturers and other entities, government organizations should also develop and organise activities, such as education and training programs, workshops, and vehicle demonstrations, to helping civics address the basic principles of automated vehicles, and the anticipated differences in the use and operation of automated vehicles from those of conventional vehicles that the public owns and operates today.

\section{CONCLUSION}

In order to better understand driver-vehicle interactions, and help improve Level 2 and Level 3 automation technology, this paper reviewed seven disengagement files reported by major companies that ran automated vehicle tests on public roads. The definition of the disengagement events focused in this study was claimed at first. Based on their different attributes, the disengagement events were classified into two types, namely PDE and ADE. The overall conditions of the disengagement reported by manufacturers were presented. Then, the main reasons of disengagement events were revealed. Among these reasons, the software issues and limitations were the most common. Cases of manufacturers'disengagements were also investigated in detail. The take-over mechanism and time reported in each company's disengagement events were discussed. Based on the analyses, recommendations to OEMs, manufacturers, and government organizations have been provided. Future work will include a comparison of the present disengagement data with those published in the following year, an investigation of driver attention and cognition when interacting with automated driving, and the development of haptic feedback HMI which considers driver cognition state.

APPENDIX

\section{NOMENCLATURE}

\begin{tabular}{cc}
\hline ADE & Active disengagement \\
ADAS & Advanced driver assistance systems \\
AI & Artificial intelligence \\
DMV & Department of motor vehicles \\
HAV & Highly automated vehicle \\
HMI & Human-machine interface \\
ISO & International Standards Organization \\
MPD & Miles per disengagement \\
NHTSA & National Highway Traffic Safety Administration \\
OEM & Original equipment manufacturer \\
PDE & Passive disengagement \\
SAE & Society of Automotive Engineers \\
TO & Take-over \\
\hline
\end{tabular}

\section{REFERENCES}

[1] F. Y. Wang, "Control 5.0: From newton to Merton in popperś cybersocial-physical spaces," IEEE/CAA J. Automat. Sin., vol. 3, no. 3, pp. 233-234, Jul. 2016.

[2] M. Da Lio, F. Biral, E. Bertolazzi, M. Galvani, P. Bosetti, D. Windridge, and F. Tango, "Artificial co-drivers as a universal enabling technology for future intelligent vehicles and transportation systems," IEEE Trans. Int. Trans. Syst., vol. 16, no. 1, pp. 244-263, Feb. 2015.

[3] L. Li, Y. S. Lv, and F. Y. Wang, "Traffic signal timing via deep reinforcement learning," IEEE/CAA J. Automat. Sin., vol. 3, no. 3, pp. 247-254, Jul. 2016.

[4] T. Qu, H. Chen, D. P. Cao, H. Y. Guo, and B. Z. Gao, "Switchingbased stochastic model predictive control approach for modeling driver steering skill," IEEE Trans. Int. Trans. Syst., vol. 16, no. 1, pp. 365-375, Feb. 2015.

[5] G. Xiong, F. H. Zhu, X. W. Liu, X. S. Dong, W. L. Huang, S H. Chen, and K. Zhao, "Cyber-physical-social system in intelligent transportation," IEEE/CAA J. Automat. Sin., vol. 2, no. 3, pp. 320-333, 2015 . 
[6] National Highway Traffic Safety Administration, Federal automated vehicles policy, Department of Transportation, pp. 1-112, Sep. 2016.

[7] SAE On-Road Automated Vehicle Standards Committee, Taxonomy and definitions for terms related to on-road motor vehicle automated driving systems," 2014.

[8] National Highway Traffic Safety Administration, "Human factors evaluation of level 2 and level 3 automated driving concepts," National Highway Traffic Safety Administration, Washington, DC, USA, Report No. DOT HS 812 044, pp. 1-300, 2014.

[9] M. Johns, B. Mok, D. M. Sirkin, N. M. Gowda, C. A. Smith, W. J. Jr. Talamonti, and W. Ju, "Exploring shared control in automated driving," in Proc. 11th ACM/IEEE International Conf. Human-Robot Interaction, Christchurch, New Zealand: IEEE, 2016, pp. 91-98.

[10] L. Lorenz, P. Kerschbaum, and J. Schumann, "Designing take over scenarios for automated driving: How does augmented reality support the driver to get back into the loop?" Proc. Human Factors Ergonomics Soc. Ann. Meet., vol. 58, no. 1, pp. 1681-1685, Sep. 2014

[11] B. Mok, M. Johns, K. J. Lee, D. Miller, D. Sirkin, P. Ive, and W. Ju, "Emergency, automation off: Unstructured transition timing for distracted drivers of automated vehicles," in Proc. 18th International Conf. Intelligent Transportation Systems, Las Palmas, Brazil: IEEE, 2015, pp. 2458-2464.

[12] C. Lv, Y. H. Liu, X. S. Hu, H. Y. Guo, D. P. Cao, and F. Y. Wang, "Simultaneous observation of hybrid states for cyber-physical systems: A case study of electric vehicle powertrain," IEEE Trans. Cybern., 2017, doi: 10.1109/TCYB.2017.2738003. (to be published)

[13] Robert Bosch, LLC, "Annual report of autonomous mode disengagements," [Online]. Available: https://www.dmv.ca.gov/ portal/ wcm/connect/ele48704-583e-4cfdaa9a-fa464cb6903d/bosch_disengagement_report.pdf?MOD=AJPERES Accessed on: Sep. 21, 2016.

[14] Delphi Automotive Systems, LLC, "Summary of autonomous vehicle disengagements," [Online]. Available: https://www.dmv. ca.gov/portal/wcm/connect/ 851838fb-da8b-45da-bd95-0ae0451de988/ 2015_Delphi_DMV_Summary.pdf?MOD=AJPERES. Accessed on: Sep. 21,2016

[15] Google Auto, "Google self-driving car testing report on disengagements of autonomous mode," [Online]. Available: https://www.dmv.ca.gov/portal/wcm/connect/dff67186-70dd-4042-bc8cd7b2a9904665/google_disengagement_report.pdf?MOD=AJPERES Accessed on: Sep. 21, 2016.

[16] Nissan North America, Inc., "Nissan autonomous vehicle disengagement report 2015," [Online]. Available: https:// www.dmv.ca. gov/portal/wcm/connect/0170b0e7-7992-45f6-918d-8d3cc5bce9ee/ nissan_disengagement_report.pdf?MOD=AJPERES.Accessed on: Dec. 23 , 2015.

[17] Mercedes-Benz Research \& Development North America, "Disengagements of autonomous vehicle system," [Online]. Available: https://www.dmv.ca. gov/portal/wcm/connect/fdea 0433-4935-414f84a2-1e815e416964/MercedezBenz_disengage_report_2016.pdf? MOD=AJPERES. Accessed on: Sep. 21, 2016.

[18] Volkswagen Group of America, "Volkswagen group of America, Inc.'s disengagement reports," [Online]. Available: https://www.dmv. ca.gov/portal/wcm/connect/9221a5cd-e67a

-4e98- aa6c-6e958c721d83/Volkswagen_disengage_report_2016.pdf? MOD= AJPERES. Accessed on: Sep. 21, 2016.

[19] Tesla Motors Inc., "Reporting disengagement of autonomous mode," [Online]. Available: https://www.dmv. ca.gov/ portal/wcm/connect/d4114e78-4c45-4328-a6b9-428e400bf067/ tesla_disengagement_report.pdf? MOD= AJPERES. Accessed on: Sep. 21, 2016.

[20] California DMV, "Final statement of reasons," [Online]. Available: https://www.dir.ca.gov/oshsb/documents/Reinforcing-Steel-ConcreteConstruction-and-Post-Tensioning-Operations-FSOR.pdf. Accessed on: Sep. 21, 2016

[21] K. Kritayakirana and J. C. Gerdes, "Autonomous vehicle control at the limits of handling," Int. J. Veh. Auton. Syst., vol. 10, no. 4, pp. 271-296, 2012.

[22] C. Urmson, J. Anhalt, D. Bagnell, C. Baker, R. Bittner, M. N. Clark, J. Dolan, D. Duggins, T. Galatali, C. Geyer, M. Gittleman, S. Harbaugh, M. Hebert, T. M. Howard, S. Kolski, A. Kelly, M. Likhachev, M.
McNaughton, N. Miller, K. Peterson, B. Pilnick, R. Rajkumar, P. Rybski, B. Salesky, Y. W. Seo, S. Singh, J. Snider, A. Stentz, W. R. Whittaker, Z. Wolkowicki, J. Ziglar, H. Bae, T. Brown, D. Demitrish, B. Litkouhi, J. Nickolaou, V. Sadekar, W. D. Zhang, J. Struble, M. Taylor, M. Darms, and D. Ferguson, "Autonomous driving in urban environments: Boss and the Urban Challenge," J. Field Rob., vol. 25, no. 8, pp. 425-466, Aug. 2008.

[23] Y. Tian, W. Y. Lo, and D. Ferguson. "Real-time active emergency vehicle detection," U.S. Patent Application No. 14/471, 640, 2014.

[24] Road vehicles-functional safety, ISO 26262-1:2011, 2011.

[25] Tesla Motors Inc., “Tesla model S-owner's manual," [Online]. Available: https://carmanuals2.com/tesla/model-s-2016-owner-s-manual89478. Accessed on: Sep. 21, 2016.

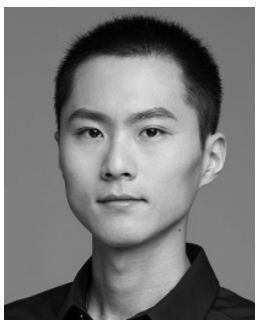

Chen Lv is currently a Research Fellow at Advanced Vehicle Engineering Center, Cranfield University, UK. He received the Ph.D. degree at Department of Automotive Engineering, Tsinghua University, China in 2016. From 2014 to 2015, he was a joint $\mathrm{Ph} . \mathrm{D}$. researcher at EECS Dept., University of California, Berkeley. His research focuses on cyberphysical system, hybrid system, advanced vehicle control and intelligence, where he has contributed over 40 papers and holds 11 granted China patents. Dr. Lv serves as a Guest Editor for IEEE/ASME Transactions on Mechatronics and IEEE Transactions on Industrial Informatics, and an Associate Editor for International Journal of Electric and Hybrid Vehicles, International Journal of Vehicle Systems Modelling and Testing, and International Journal of Science and Engineering for Smart Vehicles. He received the Highly Commended Paper Award of IMechE UK in 2012, the NSK Outstanding Mechanical Engineering Paper Award in 2014, the Tsinghua University Graduate Student Academic Rising Star Nomination Award in 2015, the China SAE Outstanding Paper Award in 2015, the 1st Class Award of China Automotive Industry Scientific and Technological Invention in 2015, and the Tsinghua University Outstanding Doctoral Thesis Award in 2016.

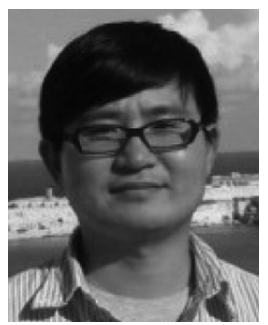

Dongpu Cao received the Ph.D. degree from Concordia University, Canada, in 2008. He is currently a Senior Lecturer at Advanced Vehicle Engineering Center, Cranfield University, UK. His research focuses on vehicle dynamics and control, automated driving and parallel driving, where he has contributed more than 100 publications and 1 US patent. He received the ASME AVTT'2010 Best Paper Award and 2012 SAE Arch T. Colwell Merit Award. Dr. Cao serves as an Associate Editor for IEEE Transactions on Intelligent Transportation Systems, IEEE Transactions on Vehicular Technology, IEEE Transactions on Industrial Electronics, IEEE/ASME Transactions on Mechatronics and ASME Journal of Dynamic Systems, Measurement, and Control. He Has Been A Guest Editor for Vehicle System Dynamics, and IEEE Transactions on Human-Machine Systems. He serves on the SAE International Vehicle Dynamics Standards Committee and a few ASME, SAE, IEEE technical committees.

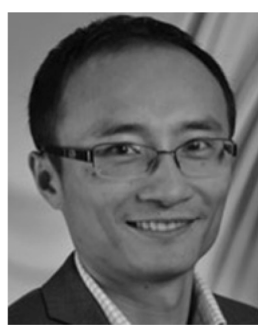

Yifan Zhao received the Ph.D. degree in automatic control and system engineering from the University of Sheffield, Sheffield, U.K., in 2007. He is currently a Lecturer in image and signal processing and degradation assessment at Cranfield University, Cranfield, U.K. His research interests include computer-visionbased process monitoring, super resolution, active thermography, and nonlinear system identification. 


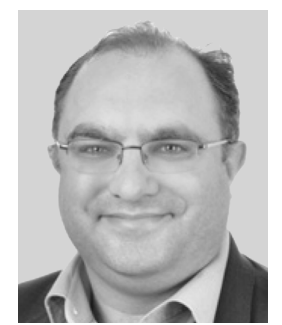

Daniel J. Auger received the M.Eng.and Ph.D. degrees in control engineering from Cambridge University, Cambridge, U.K., specializing in model validation for robust feedback control design. He is currently a Lecturer in Advanced Control and Optimization with Cranfield University, Cranfield, U.K. His recent publications have looked at techniques for addressing battery aging through optimal system design, the development of advanced battery state estimators, and techniques for the quantification of in-vehicle battery degradation. His current research interests include the application of advanced control methodologies to automotive design, development of software-embeddable battery models, optimal state-estimator design, and robust control architectures.

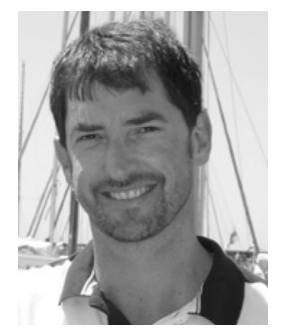

Mark Sullman is a Senior Lecturer based in the Driving Research Group, located in the Department of Advanced Systems. He received MSc and $\mathrm{PhD}$ from Massey University, New Zealand. He has more than 20 years of research and consultancy experience in the areas of occupational safety and driving behavior and has provided consultancy for a number of multinational companies. He is on the Editorial Advisor Board for Transportation Research Part F (Traffic Psychology and Behavior) and regularly reviews articles for several other international journals. In 2010 he was appointed the European representative for Division 13 of the International Association of Applied Psychology (IAAP) and has been on the Scientific Advisory board for many international conferences. He has authored over 65 journal articles, 24 book chapters, and more than 150 conference papers and industry reports.

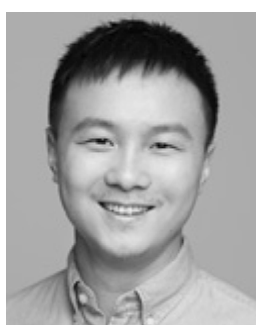

Huaji Wang received the B.S. degree in automotive engineering from Jilin University, China, in 2005 , and the Ph.D. degree in engineering from the University of Cambridge, Cambridge, U.K., in 2016, concentrating on the study of driver/vehicle systems and driver-automation collaboration. He presently works as a Research Fellow in Automated Driving at Cranfield University, UK.

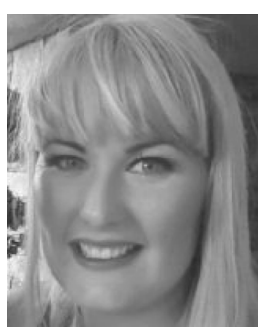

Laura Millen Dutka is a Human Machine Interface Technical Specialist at Jaguar Land Rover.

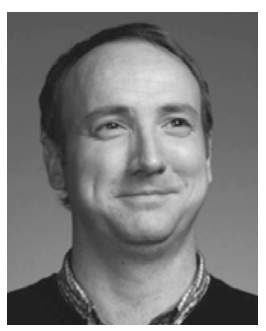

Lee Skrypchuk is a Human Machine Interface Technical Specialist at Jaguar Land Rover.

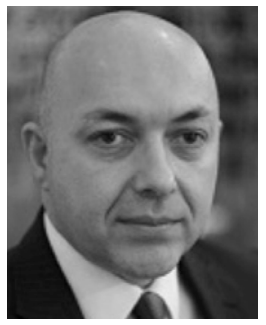

Alexandros Mouzakitis is the Head of Electrical, Electronics \& Software Engineering Research Department at Jaguar Land Rover. 
2017-12-20

\title{
Analysis of autopilot disengagements occurring during autonomous vehicle testing
}

\author{
Lv, Chen
}

IEEE

Lv C, Cao D, Zhao Y, Auger DJ, Sullman M, Wang H, Dutka LM, Skrypchuk L, Mouzakitis A, Analysis of autopilot disengagements occurring during autonomous vehicle testing, IEEE/CSS Journal of Automatica Sinica, Vol. 5, Issue 1, January 2018, pp. 58-68

http://dx.doi.org/10.1109/JAS.2017.7510745

Downloaded from Cranfield Library Services E-Repository 